\title{
Ser49Gly polymorphism in the $\beta$-adrenergic receptor 1 gene in a population sample from Rio de Janeiro state, Brazil, stratified by self-identified skin color and genetic ancestry
}

\author{
KELLY T. SANTOS ${ }^{1,2}$, ROSSANA G.A. DE FREITAS ${ }^{1,3}$, FERNANDA S.N. MANTA ${ }^{1}$, \\ ELIZEU F. DE CARVALHO ${ }^{1,4}$ and DAYSE A. SILVA ${ }^{1,4}$
}

\author{
${ }^{1}$ DNA Diagnostic Laboratory, Biology Institute, Rio de Janeiro State University, Rio de Janeiro 20550-013; \\ ${ }^{2}$ Medical Sciences Postgraduate Program, Faculty of Medical Science, Rio de Janeiro State University, Rio de Janeiro 20550-170; \\ ${ }^{3}$ Postgraduate Program in Human and Experimental Biology, Rio de Janeiro State University; ${ }^{4}$ Ecology Department, \\ Biology Institute, Rio de Janeiro State University, Rio de Janeiro 20550-013, Brazil
}

Received July 31, 2013; Accepted March 4, 2014

DOI: $10.3892 / \mathrm{mmr} .2015 .3536$

\begin{abstract}
Cardiovascular diseases (CVD) have the highest worldwide mortality rate of any type of disease. In recent years, genetic research regarding CVD has been conducted using association studies, in which the presence of a genetic polymorphism associated with a specific cell signaling pathway in a lower or in a higher frequency among patients may be interpreted as a possible causal factor. Genetic polymorphisms that occur in the $\beta$-adrenergic receptor 1 ( $\beta$-ADR1) can result in significant changes in its function that may result in physiopathologies. Ambiguous categorizations, such as skin color and self-reported ethnicity have been used in pharmacogenetic studies as phenotypic proxies for ancestry; however, admixed populations present a particular challenge to the effectiveness of this approach. The main objective of the present study was to estimate the diversity and the frequency of the Ser49Gly polymorphism of the $\beta$-ADR 1 gene in a sample of 188 male individuals from the population of Rio de Janeiro. The Ser49Gly frequencies were analyzed by two forms of sample stratification: The phenotypic criterion of black or non-black skin color, and African or non-African ancestry, defined using Y-chromosome single nucleotide polymorphisms and autosomal indel markers. These results were used to evaluate whether marker-based ancestry criteria and/or skin color were associated with the frequency of the Ser49Gly polymorphisms in the heterogeneous Rio de Janeiro/Brazilian population. The DNA fragments of interest were amplified by polymerase chain reaction with specific primers for the
\end{abstract}

Correspondence to: Dr Dayse A. Silva, DNA Diagnostic Laboratory, Biology Institute, Rio de Janeiro State University, 524 São Francisco Xavier, Maracanã, Rio de Janeiro 20550-013, Brazil

E-mail: dayse.a.silva@gmail.com

Key words: ser49gly polymorphism, $\beta-1$ adrenergic receptor gene, ancestry, population study
Ser49Gly marker, and genotyping reactions were performed by restriction with the enzyme Eco0109I. Heterozygosity values ranging from 0.25 to 0.50 and 0.20 to 0.41 were found for the groups stratified by ancestry and skin color, respectively. Using the Hardy-Weinberg equilibrium at the ser49Gly marker, it was found that there was no significant deviation in the genotype distribution of the whole Rio de Janeiro sample or the stratified sample. Analysis of the allelic distribution in the Rio de Janeiro population sample revealed frequencies of 80.30 and $19.70 \%$ for the wild-type (Ser49) and mutated (Gly49) alleles, respectively. Significant differences were observed in the allele frequencies of the Ser49Gly marker between the self-defined black and non-black phenotype, and the African and non-African descendant genotype population samples. A significant difference was also observed between blacks and African-descendant individuals, with a lesser degree of genetic differentiation. The results presented in the present study suggest that the Ser49Gly marker has a distribution that is influenced by an ancestral component, due to the increased prevalence of the Gly49 polymorphism in the black and African descendant populations of the Rio de Janeiro state. This evidence, in combination with clinical studies, may contribute to a detailed analysis of the pattern of susceptibility to CVD involved in $\beta$-ADR1 receptor mechanism failure.

\section{Introduction}

Cardiovascular diseases (CVD) have the highest mortality rate of any type of disease worldwide. In Brazil, CVD accounted for $32.3 \%$ of total mortality $(1,2)$, which justifies the search for a greater understanding of this disease. Although the associated morbidity and mortality rates remain high, treatment of CVD has progressed markedly in the past 15 years, and several drugs and procedures have been incorporated into the therapeutic strategy. This has resulted in a significant reduction in cardiovascular events with an impact on survival and quality of life, and a reduction in the costs of CVD to public and private healthcare (1). 
An important consideration in the treatment of CVD patients is the potential risk of the treatment itself. The risks associated with treatment markedly increase once various therapeutic agents at variable doses are administered together in an attempt to achieve maximum benefit. Adverse effects associated with the use of different drugs account for $>2$ million hospital admissions per year $(3,4)$. The use of $\beta$-blockers, for example, may lead to worsening symptoms of heart failure (HF). In certain studies, hypercalcemia secondary to HF treatment, was correlated with an increase in mortality from 0.03 to $0.2 \%$ and an increase of 0.24 to $1.1 \%$ hospitalizations $(3,4)$.

Therefore, despite advances in CVD therapy, it appears that the standard treatment for all patients may be inappropriate, since they do not account for the individual variability in response to drugs observed in clinical practice.

In an attempt to individualize treatment, data concerning the phenotype of the patient, such as weight and body surface area, have been considered. However, there remains significant variability in therapeutic response and clinical outcome, even in phenotypically similar patients (5-8).

Several studies have been performed in order to identify risk factors that are associated with a worse CVD prognosis and patient responses to particular treatments, that may affect current rates of morbidity and mortality $(6,7)$.

Previous studies have highlighted the importance of researching genetic factors involved in the development and treatment of various diseases. There are several studies describing the association between genomic factors and pathologies, such as right ventricular arrhythmogenic dysplasia and hypertrophic cardiomyopathy $(6,9,10)$. Genomic studies have suggested that for a considerable number of CVD patients, susceptibility to the disease is due, at least in part, to DNA polymorphisms, particularly those associated with neurotransmitter receptors (11). Since the importance of adrenergic receptors has been widely recognized, the influence of polymorphisms in these receptors and their effect on cardiovascular regulation in humans has been investigated (12).

Among the most widely investigated polymorphisms are the Arg389Gly and the Ser49Gly from the $\beta 1$-adrenergic receptor gene $(\beta$-ADR1), which have been associated with metabolic events and exhibit a causal correlation with CVD and are therefore identified as possible genetic susceptibility factors. The Arg389Arg genotype was associated with an increased adrenergic response in the acute phase of CVD and with a higher incidence of ventricular tachycardia. The genotype Ser49Ser, on the other hand, was associated with less downregulation of $\beta 1$ adrenergic receptors, resulting in an increase of the agonist expression, producing an increase in the adrenergic activity, as observed in heart failure $(12,13)$.

Other studies have demonstrated an association between the Ser49 polymorphism and lower mortality when $\beta$-blockers are used in treatment, and the Arg389 polymorphism with higher basal activity (14). This reinforces the hypothesis that the investigation of these genetic polymorphisms is an important step towards a greater understanding of the physiological changes associated with CVD.

Case-control studies can be effective in the identification of polymorphisms associated with CVD. However, the different polymorphism frequencies in samples may not always
Table I. Characteristics of the respective Rio de Janeiro sample groups genotyped for the Ser49Gly polymorphism.

\begin{tabular}{llrc}
\hline Sample group & Gender & $\mathrm{n}$ & Mean age \\
\hline Ancestry & & & \\
AFR & Male & 17 & 32 \\
NAFR & Male & 125 & 36 \\
Anc+ & & 142 & \\
Skin color & & & \\
BL & Male & 86 & 32 \\
NBL & Male & 102 & 36 \\
RJ & & 188 & \\
\hline
\end{tabular}

AFR, African descendant from Rio de Janeiro; NAFR, non-African descendant; Anc+, Rio de Janeiro sample with ancestry information; BL, black; NBL, non-black; RJ, Rio de Janeiro sample.

be associated with the underlying genetics of a particular phenotype; they can also be derived from population stratification (15).

In this case, if the prevalence of this candidate gene is particularly high in a particular ethnic group and if that group is over-represented among cases compared with the controls, the frequency of this allele in this group may be mistakenly associated with the disease in question featuring an erroneous association (16). In addition, a more frequent allele in this group may be erroneously associated with the disease in question. These spurious associations may be due to the genetic stratification of the population analyzed and often cannot be perceived by information provided only by the object of study. This means that if a sample is stratified based on their ancestry, for example, this stratification cannot be detected through self ethnic statement or color self-declaration, information commonly available in clinical studies. Therefore, ancestry genetic markers should be used in order to investigate ancestry background (15). This error is evident when admixed populations, such as the Brazilian population, are considered. Since skin color and self-reported ethnicity have been used in pharmacogenetics as phenotypic proxies for ancestry, population admixture presents questions regarding the effectiveness of this approach. In the present study, estimates of ancestry were important strategies in order to minimize the effect of the genetic population substructure.

Although frequency data concerning polymorphisms of $\beta$-ADR1 for different ethnic groups are well-reported in European, North American and Asian populations, data regarding the Brazilian population are limited, particularly with regard to the size of each ethnic group component in this admixed population. The aim of the present study was to analyze the Ser49Gly polymorphism in the $\beta$-ADR1 receptor gene, comparing allele and genotype frequencies in a sample from the population of the Rio de Janeiro state, Brazil. The frequencies were stratified by the phenotypic criterion of black and non-black skin color, and by African and non-African genetic ancestry, defined by Y-chromosome single nucleotide polymorphisms (SNPs) and autosomal indel markers (AIMs), to evaluate whether the marker-based ancestry criterion and/or skin color are associated with Ser49Gly polymorphisms in the heterogeneous Rio de Janeiro Brazilian population. 
Table II. Allelic and genotypic frequencies in Rio de Janeiro sample grouped by ancestry and diversity parameters (homozigosity and heterozigosity).

\begin{tabular}{lccc}
\hline Genetic information & Anc+ $(\mathrm{n}=142)$ & AFR $(\mathrm{n}=17)$ & NAFR (n=125) \\
\hline Allele frequency & & & 55.90 \\
Ser49 (\%) & 81.70 & 44.10 & 85.20 \\
Gly49 (\%) & 8.30 & & 14.80 \\
Genotypic frequency & & 11.80 & 72.80 \\
Ser/Ser (\%) & 65.40 & 88.20 & 26.40 \\
Ser/Gly (\%) & 33.80 & 0.00 & 0.80 \\
Gly/Gly (\%) & 0.80 & 0.882 & 0.280 \\
Ho & 0.352 & 0.508 & 0.253 \\
He & 0.300 & 0.002 & 0.307 \\
P-value & 0.046 & & \\
\hline
\end{tabular}

Anc+, Rio de Janeiro sample with ancestry information; AFR, African descendant from Rio de Janeiro; NAFR, non-African descendant; Ho, homozigosity; He, heterozigosity.

\section{Materials and methods}

Study population. A total of 188 DNA samples from genetically unrelated individuals born in the state of Rio de Janeiro were collected by the DNA Diagnostic Laboratory of Rio de Janeiro State University (Rio de Janeiro, Brazil). The samples were collected by informed voluntary donation for research purposes and was approved by the Local Institutional Research and Ethics Committee at Rio de Janeiro State University, according the process number CAAE:0067.0.228.000-09 and written informed consent was obtained from all subjects. For the analyses, subjects were grouped by skin color as black-Rio de Janeiro (BL, $n=86$ ) or non-black (NBL, $n=102)$, and by genetic ancestry confirmed by analysis of AIMs $(17,18)$ and Y-chromosome SNPs (19) as African descendant (AFR, n=17) or non-African descendant (NAFR, $n=125$ ) (Table I).

Blood collection. Samples of peripheral venous blood were obtained with fully informed consent. The samples were collected in Vacutainer tubes with EDTA anticoagulant and maintained at $4^{\circ} \mathrm{C}$ until the DNA was extracted using the salting out method (20).

Polymerase chain reaction (PCR) analysis. PCR was performed using 50 ng DNA, $2.5 \mathrm{mM}$ deoxyribonucleotide triphosphate, $4 \mathrm{X}$ buffer containing $50 \mathrm{mM} \mathrm{KCl}, 4 \mathrm{mM} \mathrm{MgCl}_{2}, 10 \mathrm{mM}$ Tris-HCl (pH 8.5), 1.0 IU TaqDNA polymerase and $1 \mu \mathrm{l}$ of each primer for the Ser49Gly polymorphism. The primer sequences were as follows: Sense: 5'-CCGGGC TTCTGGGGTGTTCC-3' and antisense: 5'-GGCGAGGTGA TGGCGAGGTAGC-3'. The cycling conditions were: $94^{\circ} \mathrm{C}$ for $3 \mathrm{~min}, 40$ cycles of $94^{\circ} \mathrm{C}$ for $30 \mathrm{sec}, 65^{\circ} \mathrm{C}$ for $30 \mathrm{sec}$, and $72^{\circ} \mathrm{C}$ for $45 \mathrm{sec}$ with a final extension of $72^{\circ} \mathrm{C}$ for $7 \mathrm{~min}$. The PCR-amplified products were separated by electrophoresis on $2 \%$ agarose gel.

The PCR products were digested with 1X NEB04 buffer and 1 unit Eco0100I restriction endonuclease (New England Biolabs, Beverly, MA, USA), with band fragments of $345 \mathrm{bp}$ (Ser/Gly) and 219 bp (Gly/Gly). Following digestion, the genotypes were separated and identified by gel electrophoresis in $2.0 \%$ agarose or $8 \%$ polyacrylamide gel.
Statistical analysis. Estimates of genetic diversity, allele and genotype frequencies, population differentiation and genetic distance using the fixation index (Fst) measure were computed using the Arlequin program, version 3.5 (21).

\section{Results}

Characterization of population sample. The Ser49Gly polymorphism was analyzed in 188 individuals, grouped by skin color as black (BL) or non-black (NBL). Out of the 188 individuals, 142 were also grouped by ancestry defined by Y-chromosome SNPs and AIMs as African descendant (AFR) or non-African descendant (NAFR). Table I shows the characteristics of the groups.

Heterozygocity, genotype and allele frequencies. The allele and genotype frequencies, genetic diversity, expected and observed heterozygosity values and significance (P-value) for the Ser49Gly polymorphism in the $\beta$-ADR1 gene were calculated using the Hardy-Weinberg equation for the population samples grouped by ancestry (Table II) and skin color (Table III).

The values of heterozygosity ranged from 0.25 to 0.50 and 0.20 to 0.41 in the groups stratified by ancestry and skin color, respectively. Using the Hardy-Weinberg equilibrium, it was found that there was no significant deviation from this marker genotype distribution $(\mathrm{P}>0.05)$ in the Rio de Janeiro sample (RJ) or very close to the established statistical significance level of $\mathrm{p}=0.05$ in Anc+ (Tables II and III).

The distribution of alleles in AC_RJ reveals frequencies of 80.30 and $19.70 \%$ for the wild-type (Ser49) and mutated (Gly49) alleles, respectively. The same pattern was observed in the Anc+ group (Table II).

Comparison with other world population samples. The Rio de Janeiro state population sample and the groups stratified by skin color or genomic ancestry were also compared with other world populations based on the estimated index of Wright's F-statistics (Fst). This index is calculated using the variance of allele frequencies between pairs of samples and converted 
Table III. Allelic and genotypic frequencies in the Rio de Janeiro sample grouped by skin color and diversity parameters (homozigosity and heterozigosity).

\begin{tabular}{lccc}
\hline Genetic information & RJ $(\mathrm{n}=188)$ & $\mathrm{BL}(\mathrm{n}=86)$ & $\mathrm{NBL}(\mathrm{n}=102)$ \\
\hline Allele frequency & & & 70.90 \\
Ser49 (\%) & 80.30 & 29.10 & 88.20 \\
Gly49 (\%) & 19.70 & & 11.80 \\
Genotypic frequency & & 47.70 & 77.50 \\
Ser/Ser (\%) & 63.80 & 50.00 & 21.60 \\
Ser/Gly (\%) & 34.60 & 2.30 & 0.90 \\
Gly/Gly (\%) & 1.60 & 0.535 & 0.216 \\
Ho & 0.362 & 0.415 & 0.209 \\
He & 0.317 & 0.008 & 1.000 \\
P-value & 0.062 & & \\
\hline
\end{tabular}

BL, black individuals from Rio de Janeiro; NBL, non-black; RJ, Rio de Janeiro population-accumulated data; Ho, homozigosity; He, heterozigosity.

Table IV. Statistical analysis (Fst) in Rio de Janeiro population samples grouped by ancestry and skin color. Below the diagonal are shown the P-values and above Fst values.

\begin{tabular}{lllllrr}
\hline Group & BL & NBL & AFR & NAFR & RJ \\
\hline BL & 0.000 & 0.085 & 0.033 & 0.055 & 0.020 & 0.028 \\
NBL & 0.000 & 0.000 & 0.286 & 0.000 & 0.018 & 0.012 \\
AFR & 0.040 & 0.000 & 0.000 & 0.222 & 0.140 & 0.159 \\
NAFR & 0.000 & 0.385 & 0.000 & 0.000 & 0.004 & 0.000 \\
RJ & 0.006 & 0.014 & 0.000 & 0.257 & 0.671 & 0.002 \\
Anc+ & 0.002 & 0.042 & 0.000 & 0.000 \\
\hline
\end{tabular}

BL, black individuals from Rio de Janeiro; NBL, non-black; AFR, African descendant; NAFR, non-African descendant; RJ, Rio de Janeiro population-accumulated data; and Anc+, Rio de Janeiro population-accumulated data with ancestry information.

into a pattern of genetic distance. Fst values are presented in Tables IV and V and the statistical significance of the analysis is presented in Table VI. The largest genetic distances were observed between the AFR and NBL groups ( $F s t=0.286$, $\mathrm{P}<0.05)$ followed by the AFR and NAFR groups $(\mathrm{Fst}=0.222$, $\mathrm{P}>0.05)$.

\section{Discussion}

The construction and analysis of databases of allele frequencies in population studies can be addressed to research applications of quantitative traits in association studies (22). Populations that have different characteristics among groups of individuals may exhibit differences in allele frequencies, which may result in spurious associations between a marker and a particular phenotype. The correct interpretation of the data generated from population studies may decrease this type of occurrence (15).

In the present study, the frequencies of the Ser49Gly polymorphism in the $\beta$-ADR1 gene were estimated from a sample of 188 individuals of the population of Rio de Janeiro. The frequencies were also analyzed by sample stratification using either the phenotypic criterion of black (BL) or non-black (NBL) skin color, or by genetic ancestry, defined by ancestry information markers as African descendant (AFR) or non-African descendant (NAFR). The frequencies obtained in the present study were also compared with those of other world populations in order to evaluate whether there were significant differences in the frequency of the studied marker in different populations and whether the criteria of ancestry and/or skin color determine the pattern of differentiation and genetic distance.

The observed heterozygosity was higher than expected; however, this difference may be due to chance, since no deviation from the Hardy-Weinberg equilibrium was identified.

The distribution of the alleles from the 188 individuals revealed that the wild-type allele Ser49 has a frequency of $80.30 \%$ compared with a frequency of the mutant allele Gly49 of $19.70 \%$. The same pattern was observed for the other analyzed subgroups, stratified by ancestry (Table II) or skin color (Table III). Studies in different world populations, including European (23-26), Asian $(27,28)$ and other Latin American populations (29) also revealed higher frequencies of the Ser49 wild allele compared with the Gly49 allele.

The analysis of the distribution genotypes of the whole sample revealed the occurrence of three genotypes, with the greatest frequency of the wild homozygous Ser49Ser in the NAFR (Table II) and NBL (Table III) groups. In addition, 

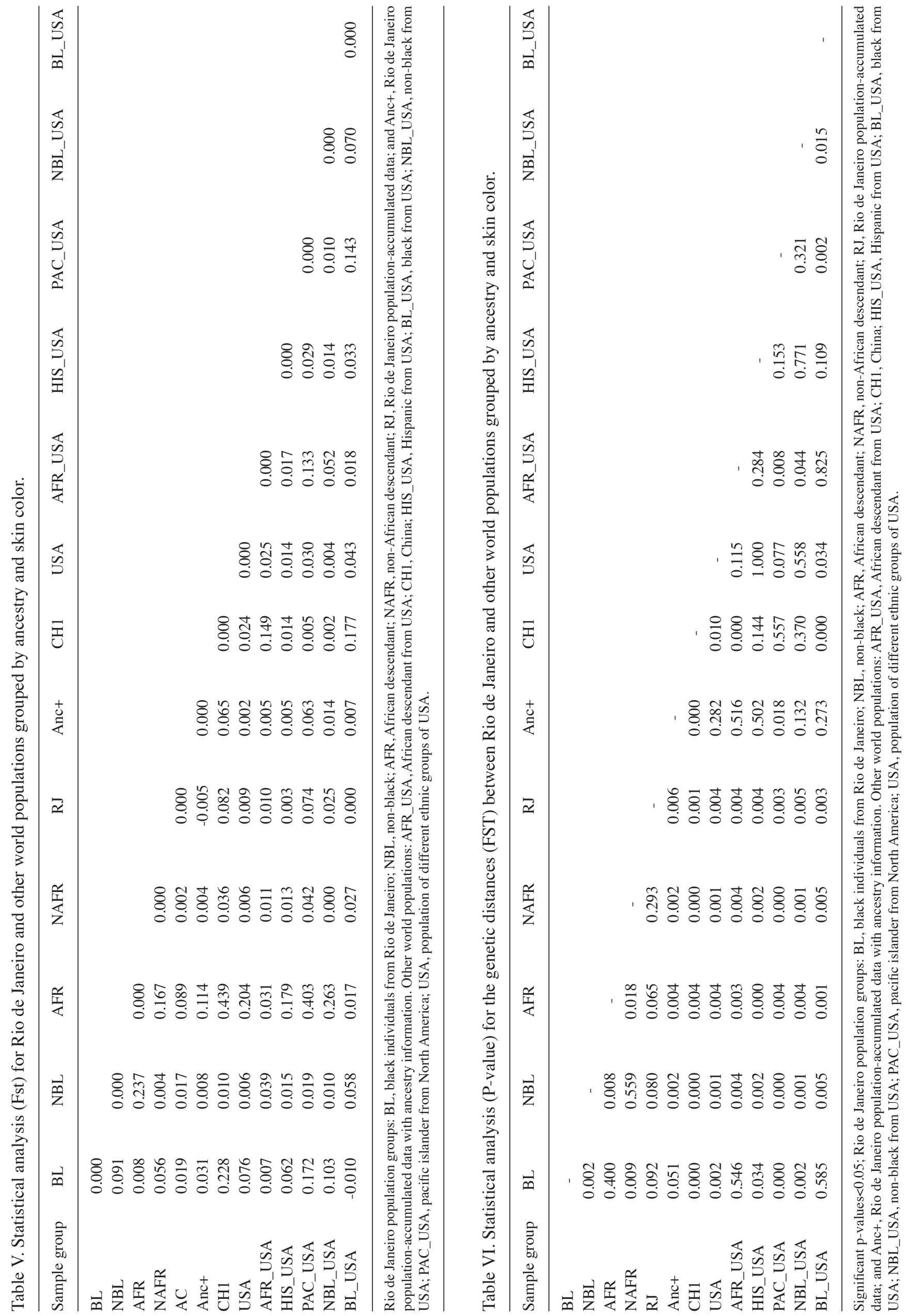
increases in the frequency of heterozygous Ser49Gly and homozygous Gly49Gly mutant genotypes among AFR (Table II) and BL (Table III) groups were observed.

A study by Ramu et al (27), using a sample of healthy individuals from India together with data collected from other populations (China, Japan, Mexico and Sweden), has revealed that the frequency of genotype Ser49Ser was also increased. Furthermore, the increase of heterozygous and homozygous mutants was also observed in a sample of individuals of African descent, consistent with the results of the present study. A similar result was observed by Kurnik et al (30) by investigating individuals of African- and non-African descent in North America, using skin color as a parameter. A lower frequency of the homozygous Ser/Ser genotype with a consequently high frequency of the Ser/Gly and Gly/Gly genotypes was identified in the African descendent population.

Populations are never pure in a genetic sense. Therefore, defining boundaries between individuals or populations based on genetic criteria is inappropriate. Nevertheless, individuals commonly form groups according to geographical distance or shared cultures (31). These groups are subject to factors, such as isolation, genetic drift, selection and migration that may influence the distribution of genetic markers, guiding a pattern of differentiation among populations. By consideration of these factors, the variation in the pattern of distribution of alleles among populations can be used as an estimate of the genetic distance between such groups. Jorde and Wooding (31), used Fst to evaluate genetic differentiation, measuring the proportion of the total variance of allelic frequencies that occurred between subpopulations.

In the present study, the largest genetic distances, as determined by the analysis of the Ser49Gly marker, were identified between the AFR_RJ and the NBL_RJ groups (Fst=0.286 and $\mathrm{P}<0.05)$, followed by the AFR_RJ and NAFR_RJ groups (Fst $=0.222$ and $P>0.05$ ). This result is in accordance with the findings of studies in which the highest measures of genetic distance among African populations occurred in association with the non-African populations (31).

The analysis of Fst in the present study also revealed that the closest genetic distance was observed between the NBL_RJ and AFR_RJ groups with P>0.05. However, significant distances were observed between the BL_RJ and AFR_RJ groups $(\mathrm{P}<0.05)$. These data suggest that there is a greater correlation between the sample data stratified by ancestry and skin color in relation to non-African inheritance when compared with African inheritance.

The results also suggest that the grouping of individuals characterized by skin color should be determined by an analysis of the contribution of the non-African ancestral component. Studies that use samples of individuals characterized only on the skin color criterion, disregarding information with regard to the ancestry of the individual, may have results shift significantly due to this spurious association.

In the present study, the genetic distances in a sample from the state of Rio de Janeiro, stratified by skin color and genetic ancestry, were compared with other world populations, using the pattern of Fst (Table V). The non-black and non-African descendant individuals in the present study were similar to the admixed populations of other world locations, indicating that these groups share similar non-African ancestry. The populations of Asian ethnicity exhibited a distribution of allelic polymorphisms of the receptor $\beta$-ADR1 in accordance with those observed in the literature for these populations (27-29). These results demonstrate that cultural and geographical barriers are strong components for the differentiation pattern of genetic flow.

With the realization of the differences in the distribution of alleles in different world populations of the Ser49Gly marker, a question arises regarding the differing response sensitivity of the $\beta$-ADR receptors in CVD due to ethnic differences, and whether this knowledge can contribute to improved characterization of the physiopathology of this disease.

In the clinical context, certain studies analyzing $\beta$-blocker treatment in cardiac patients from different ethnic groups revealed differences in sensitivity to the drug and the time to achieving effective clinical treatment. For example, in a study of the different ethnic groups in Malaysia (Chinese, Indian and Malay), the Chinese ethnic group was less sensitive following stress tests to the antihypertensive effects of propranolol compared with the Indian and Malay ethnic groups (9). Two further studies investigating the association between ancestral information and clinical treatment outcomes found that in individuals of African descent, the effectiveness of propranolol treatment improved with dose increases when compared with individuals of non-African descent $(32,33)$. This suggests that detailed knowledge of individuals through analysis of ancestry can optimize therapeutic conditions, with a consequent reduction in the cost of treatment.

In the context of genetic analysis, a study by Kurnik et al (30), found a positive association between mutated alleles and the lowest level of response to atenolol treatment as determined by electrocardiograms and analyzing the area under the curve. In an analysis of 144 patients with systolic heart failure, a positive association was observed between the polymorphism Ser49Gly and atrial fibrillation (12). In another study conducted to understand the physiological changes associated with dobutamine administration, 38 healthy subjects underwent tests of heart rate, systolic and diastolic blood pressure, and a physiological improvement was observed between the Ser49Gly polymorphism and cardiovascular physiology with the use of $\beta$-blocker (23).

The results of the present study suggest that the Ser49Gly marker has a distribution influenced by an ancestral component, due to a higher prevalence of the Gly49 polymorphism in the black and African descendant populations of Rio de Janeiro. This validates the hypothesis that ancestral knowledge is an important tool to reduce the likely bias observed in association studies where ethnicity is self-defined, particularly in admixed populations thus resulting in potential failure in the characterization of samples. Such evidence, in combination with clinical studies, may contribute to a detailed analysis of the pattern of susceptibility to CVD with $\beta$-ADR 1 receptor mechanism failure. This may enable indication of appropriate therapy for each individual, thus contributing to an improved prognosis of disease in the wider population.

\section{Acknowledgements}

Financial support was granted by the Carlos Chagas Filho Foundation Research Support of the State of Rio de Janeiro. 


\section{References}

1. Rivera ARQ, Carvalho CCF, Arantes MK, Franchetti M, Silva MMF and Alcaraz ZGM: Proteína C-Reativa e Doença Arterial Coronária. Souza GMR, Piegas LS and Souza JENR (eds). Série Monografias Dante Pazzanese, Rio de Janeiro, pp1-39, 2004.

2. Furukawa TS, Santo AH and Mathias TAF: Multiple causes of death related to cerebrovascular diseases in the State of Parana, Brazil. Rev Bras Epidemiol 14: 231-239, 2011.

3. Metzger I, Souza-Costa DC and Tanus-Santos JE: Pharmacogenetics: Principles, Applications and Perspectives. Medicina in Ribeirão Preto 39: 515-521, 2006 (In Portuguese) Available at http://revista.fmrp.usp.br/2006/vol39n4/1_farmacogenetica_principios_aplicacoes_perspec.pdf.

4. Juurlink DN, Mamdani MM, Lee DS, Kopp A, Austin PC, Laupacis A and Redelmeier DA: Rates of hyperkalemia after publication of the Randomized Aldactone Evaluation Study. N Engl J Med 351: 543-551, 2004.

5. Howlett JG, Mckelvie RS, Costigan J, et al: The 2010 Canadian Cardiovascular Society guidelines for the diagnosis and management of heart failure update: heart failure in ethnic minority populations, heart failure and pregnancy, disease management, and quality improvement/assurance programs. Can J Cardiol 26: 185-202, 2010.

6. McNamara DM: Emerging role of pharmacogenomics in heart failure. Curr Opin Cardiol 23: 261-268, 2008.

7. Rasool AH, Rahman AR, Ismail R, Hatim S, Adbullah AR, Singh $\mathrm{R}$ and Haron R: Ethnic differences in response to non-selective beta-blockade among racial groups in Malaysia. Int J Clin Pharmacol Ther 38: 260-269, 2000.

8. Taylor, JS and Ellis GR: Racial differences in responses to drug treatment: implications for pharmacotherapy of heart failure. Am J Cardiovasc Drugs 2: 389-399, 2002.

9. Arking DE and Chakravarti A: Understanding cardiovascular disease through the lens of genome-wide association studies. Trends Genet 25: 387-394, 2009.

10. Carson P, Ziesche S, Johnson G and Cohn JN: Racial differences in response to therapy for heart failure: analysis of the vasodilator-heart failure trials. Vasodilator-Heart Failure Trial Study Group. J Card Fail 5: 178-187, 1999.

11. Esler M, Kaye D, Lambert G, Esler D and Jennings G: Adrenergic nervous system in heart failure. Am J Cardiol 80: 7L-14L, 1997.

12. Nascimento BC, Pereira SB, Ribeiro GS and Mesquita ET: Beta1-adrenergic receptor polymorphisms associated with atrial fibrillation in systolic heart failure. Arq Bras Cardiol 98: 384-389, 2012 (In English and Portuguese).

13. Pereira SB, Velloso MW, Chermont S, et al: $\beta$-adrenergic receptor polymorphisms in susceptibility, response to treatment and prognosis in heart failure: implication of ethnicity. Mol Med Rep 7: 259-265, 2013.

14. Small KM, Wagoner LE, Levin AM, Kardia SL and Liggett SB: Synergistic polymorphisms of beta1- and alpha2C-adrenergic receptors and the risk of congestive heart failure. $\mathrm{N}$ Engl J Med 347: 1135-1142, 2002.

15. Tian C, Gregersen PK and Seldin MF: Accounting for ancestry: population substructure and genome-wide association studies. Hum Mol Genet 17: R143-R150, 2008.

16. Wang C, Zhan X, Bragg-Gresham B, et al: Ancestry estimation and control of population stratification for sequence-based association studies. Nat Genet 46: 409-415, 2014
17. Manta FS, Pereira R, Caiafa A, Silva DA, Gusmão L and Carvalho EF: Analysis of genetic ancestry in the admixed Brazilian population from Rio de Janeiro using 46 autosomal ancestry-informative indel markers. Ann Hum Biol 40: 94-98, 2013.

18. Pereira R, Phillips C, Pinto N, et al: Straightforward inference of ancestry and admixture proportions through ancestry-informative insertion deletion multiplexing. PLoS One 7: e29684, 2012.

19. Silva DA, Carvalho E, Costa G, Tavares L, Amorim A and Gusmão L: Y-chromosome genetic variation in Rio de Janeiro population. Am J Hum Biol 18: 829-837, 2006.

20. Miller SA, Dykes DD and Polesky HF. A simple salting out procedure for extracting DNA from human nucleated cells. Nucleic Acids Res 16: 1215, 1988.

21. Excoffier L, Laval G and Schneider S: Arlequin (version 3.0): an integrated software package for population genetics data analysis. Evol Bioinform Online 1: 47-50, 2007.

22. Salazar-Flores J, Dondiego-Aldape R, Rubi-Castellanos R, et al: Population structure and paternal admixture landscape on present-day Mexican-Mestizos revealed by Y-STR haplotypes. Am J Hum Biol 22: 401-409, 2010.

23. Kindermann M, Seeland U, Ruhnke P, Böhm M and Maack C: Functional effects of $\beta_{1}$-adrenoceptor polymorphisms on the hemodynamic response to dobutamine with and without $\beta$-blocker administration. Clin Res Cardiol 100: 129-137, 2011.

24. Nia AM, Caglayan E, Gassanov N, et al: Beta1-adrenoceptor polymorphism predicts flecainide action in patients with atrial fibrillation. PLoS One 5: e11421, 2010.

25. Nieminen T, Lehtimäki T, Laiho J, et al: Effects of polymorphisms in betal-adrenoceptor and alpha-subunit of $G$ protein on heart rate and blood pressure during exercise test. The Finnish Cardiovascular Study. J Appl Physiol 100: 507-511, 2006.

26. Bengtsson K, Melander O, Orho-Melander M, Lindblad U, Ranstam J, Råstam L and Groop L: Polymorphism in the beta(1)-adrenergic receptor gene and hypertension. Circulation 104: 187-190, 2001.

27. Ramu P, Mahesh Kumar KN, Shewade DG, Swaminathan RP, Dutta TK, Balachander J and Adithan C: Polymorphic variants of beta1 adrenergic receptor gene (Ser49Gly \& Arg389Gly) in healthy Tamilian volunteers. Indian J Med Res 132: 62-66, 2010.

28. Nonen S, Okamoto H, Akino M, et al: No positive association between adrenergic receptor variants of alpha2cDel322-325, beta1Ser49, beta1Arg389 and the risk for heart failure in the Japanese population. Br J Clin Pharmacol 60: 414-417, 2005.

29. Zaroff JG, Pawlikowska L, Miss JC, et al: Adrenoceptor polymorphisms and the risk of cardiac injury and dysfunction after subarachnoid hemorrhage. Stroke 37: 1680-1685, 2006.

30. Kurnik D, Li C, Sofowora GG, et al: Beta-1-adrenoceptor genetic variants and ethnicity independently affect response to beta-blockade. Pharmacogenet Genomics 18: 895-902, 2008.

31. Jorde LB and Wooding SP: Genetic variation, classification and 'race'. Nat Genet 36 (11 Suppl): S28-S33, 2004.

32. Johnson JA: Racial differences in lymphocyte beta-receptor sensitivity to propranolol. Life Sci 53: 297-304, 1993.

33. Johnson JA, Burlew BS and Stiles RN: Racial differences in beta-adrenoceptor-mediated responsiveness. J Cardiovasc Pharmacol 25: 90-96, 1995 